\title{
Characterization of the Polarization State of Embedded Piezoelectric Transducers by Thermal Waves and Thermal Pulses
}

\author{
A. Eydam ${ }^{1}$, G. Suchaneck ${ }^{1}$, G. Gerlach ${ }^{1}$ \\ ${ }^{1}$ Technische Universität Dresden, Solid State Electronics Laboratory, 01062 Dresden, Germany \\ agnes.eydam@tu-dresden.de
}

\begin{abstract}
In this work, we demonstrate that thermal methods are a promising approach for non-destructive characterization of the polarization state of embedded piezoelectric transducers in integrated sensoractuator modules. The modules are subjected to periodic heating by a square-wave modulated laser beam or to single-pulse heating by a laser pulse. The frequency or time dependence of the resulting pyroelectric current carries information about the pyroelectric coefficient depth profile which correlates directly with the polarization distribution. In frequency domain, the sample behavior is described by a harmonically heated piezoelectric plate exhibiting heat losses to the environment characterized by a continuous distribution of relaxation times. This analytical model enables additionally the evaluation of the homogeneity of the polarization.
\end{abstract}

Key words: piezoelectric transducer, polarization state, laser intensity modulation method, thermal pulse method, relaxation time

\section{Introduction}

Piezoelectric transducers are embedded into structural components to make them controllable or responsive to their environment. The thereby created smart structures find applications for instance for health-monitoring of safety components, for reducing noise emission in automobile engineering, for damping vibrations etc. Their mass-production requires control of the polarization state due to mechanical and thermal loads appearing during device fabrication.

Non-destructive methods for obtaining polarization profiles rely on an external excitation of the material leading to a local change of material properties [1]. A thermal excitation in terms of thermal waves or thermal pulses gives rise to a pyroelectric current which carries information on the polarization profile. In frequency domain, the Laser Intensity Modulation Method (LIMM) is well-established. Whereby thermal waves are generated by a periodically modulated laser beam [2]. When thermal pulses are applied with a pulsed laser, the signal is recorded in time domain [3].

In this work, we apply both methods to evaluate the polarization state of lead-zirconate-titanate (PZT) plates or fibers integrated in lowtemperature-cofired-ceramics (LTCC) and epoxy resin.

\section{Experimental Methods}

Two different types of embedded piezoelectric transducers were evaluated:

(i) A LTCC/PZT sensor and actuator consisting of an already sintered PZT plate (CeramTec Sonox ${ }^{\circledR}$ P53) with a size of $25 \times 10 \times 0.2 \mathrm{~mm}^{3}$ embedded in the center of a sintered LTCC module $(45 \times 20 \times$ $0.7 \mathrm{~mm}^{3}$, Heraeus HeraLock $\circledast$ TapeHL2000). Sample fabrication is described in detail elsewhere [4]. The sample capacitance was $30 \mathrm{nF}$, the dielectric loss tangent amounted to about $2 \%$ at $10 \mathrm{kHz}$.

(ii) A commercial M-8528-P2 macro-fiber composite (MFC) actuator (Smart Materials, Dresden, Germany) with an overall length of $105 \mathrm{~mm}$, an active length of $85 \mathrm{~mm}$, an active width of $28 \mathrm{~mm}$, a thickness of about $0.3 \mathrm{~mm}$, a sample capacitance of $170 \mathrm{nF}$ and a dielectric loss tangent of about $5 \%$ at $10 \mathrm{kHz}$. PZT macrofibers are embedded in epoxy resin. They are electrically contacted by copper electrode strips and covered by a Kapton film.

In comparison, a non-embedded PZT plate with a size of $(10 \times 7 \times 0.2) \mathrm{mm}^{3}$ covered by a nickel-chromium electrode was analysed. 
For LIMM measurements, the samples were periodically heated by an array of six laser diodes or a single laser diode (LCU98A041A, Laser Components $\mathrm{GmbH}$, Olching, Germany) square-wave-modulated with frequencies of up to $1 \mathrm{kHz}$ each with a power of $14 \mathrm{~mW}$ at a wavelength of $980 \mathrm{~nm}$. The complex pyroelectric current was determined by an impedance/gain-phase analyzer (Solartron 1260, Solartron Analytical, Farnborough, UK) with DC coupling. In order to reduce noise, 30 measurement repetitions were used for averaging.

Thermal pulse measurements were carried out by heating the samples with a pulsed laser diode (LC905D1S3J09UA, Laser Components $\mathrm{GmbH}$, Olching, Germany) at a wavelength of $905 \mathrm{~nm}$ with a peak power of maximal $75 \mathrm{~W}$, a pulse width between 150 and 1000 ns and a repetition frequency between $1 \mathrm{~Hz}$ and $1 \mathrm{kHz}$. The pyroelectric current was transformed to a voltage by a current amplifier (DLPCA-200, Femto Messtechnik $\mathrm{GmbH}$, Berlin, Germany) and the signal was recorded by a Waverunner ${ }^{\circledR}$ Xi-A oscilloscope (LeCroy, Chestnut Ridge, USA).

\section{Theory}

The pyroelectric current spectrum obtained by LIMM is described in general by the basic LIMM equation [5]:

$I(\omega)=\frac{A}{d} \int_{0}^{d} p(z) \frac{\partial}{\partial t} T(\omega, z, t)$,

where $A$ is the heated area, $d$ the piezoelectric film thickness, $p(z)$ the pyroelectric coefficient distribution and $T$ the temperature change relative to the environment. Considering a harmonically heated piezoelectric plate, the pyroelectric response yields [5]:

$$
\begin{array}{r}
I_{\sim}(\omega)=\frac{\Phi_{0} A}{c \rho \cdot d}\left(p_{0} \frac{i \omega \tau_{t h}}{1+i \omega \tau_{\text {th }}}+\right. \\
\\
\left.\sum_{n=1}^{\infty} p_{n} \frac{i \omega \tau_{d} / n^{2}}{1+i \omega \tau_{d} / n^{2}}\right),
\end{array}
$$

where $\Phi_{0}$ is the heat flux absorbed by the plate surface, $c$ the specific heat, $\rho$ the density, $p_{0}$ the average pyroelectric coefficient, $p_{\mathrm{n}}$ the spatially dependent parts of the pyroelectric coefficient and $\tau_{d}=d^{2} / \pi^{2} a$ the heat diffusion time with the thermal diffusivity $a$. The thermal relaxation time $\tau_{\text {th }}$ characterizes the heat losses of the PZT plate to the environment, in our case to the structural components.
For a continuous distribution of relaxation times instead of a single time constant and a homogeneous polarization with $p_{\mathrm{n}}=0$, eq. (2) results in [6]:

$$
I(\omega)=\frac{\Phi_{0} A}{c \rho \cdot d} p_{0}\left[1-\frac{1}{\left.1+\left(i \omega \tau_{t h}\right)^{\alpha}\right]^{\beta}}\right],
$$

This model is equivalent to the HavriliakNegami function as known from dielectric relaxation with the empirical parameters $\alpha$ and $\beta$ accounting for the broadness and asymmetry of the distribution function. Two special cases are the Cole-Cole relaxation for $\beta=1$ and $\alpha<1$ and the Cole-Davidson relaxation for $\alpha=1$ and $\beta<1$.

A superposition of several relaxation processes is also possible, depending on the sample design.

The pyroelectric current acquired by the thermal pulse method is in general also characterized by eq. (1), but without the frequency dependence.

\section{Results and Discussion Laser Intensity Modulation Method}

Fig. 1 illustrates the pyroelectric current spectrum of a LTCC/PZT module fitted to eq. (2). Between $0.1 \mathrm{~Hz}$ and $10 \mathrm{~Hz}$ the embedded PZT plate loses heat to the LTCC layers with a thermal relaxation time of $0.16 \mathrm{~s}$. The minor decrease of the real part at higher frequencies is caused by a slightly inhomogeneous polarization distribution which is taken into account by $p_{1}=-p_{0} / 5$. A detailed analysis was reported in [7].

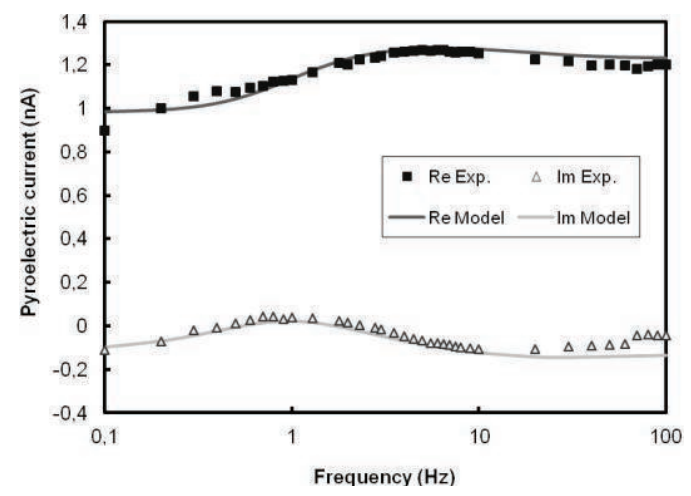

Fig. 1. Pyroelectric current spectrum of a LTCC/PZT sensor-actuator in comparison to a fit to eq. (2) with $p_{0}$ and $p_{1}$.

The MFC actuator is an example for a broad relaxation time distribution which is well described by a Cole-Cole function with $\alpha=0.4$ (fig. 2). The relaxation time constant with a value of $5.3 \mathrm{~s}$ describes the heat loss of the 
sample surface to the environment with a thermal conductance of about $100 \mathrm{~W} / \mathrm{m}^{2} \mathrm{~K}$. Here, the pyroelectric current spectrum is satisfactorily modeled by assuming a homogeneous polarization.

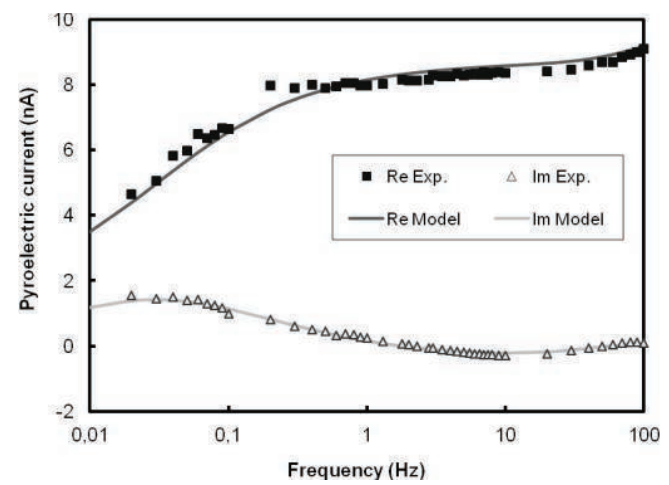

Fig. 2. Pyroelectric current spectrum of a MFC actuator in comparison to a fit to eq. (3).

The non-embedded PZT plate was measured in comparison to the embedded samples. The analytical model in Ref. 5 was originally developed for such samples where only the interface sample-air is present. The obtained pyroelectric spectrum that is illustrated in fig. 3 is comparable to the results in Ref. 5. The thermal relaxation time amounts to $0.8 \mathrm{~s}$. The fit is not an ideal Debye-like model but there is a slight time distribution which is attributed to the influence of the electrodes and the electrical contact by a wire. The spectrum is best described by a Cole-Davidson relaxation with $\beta=0.9$. The PZT plate shows a homogeneous polarization distribution.

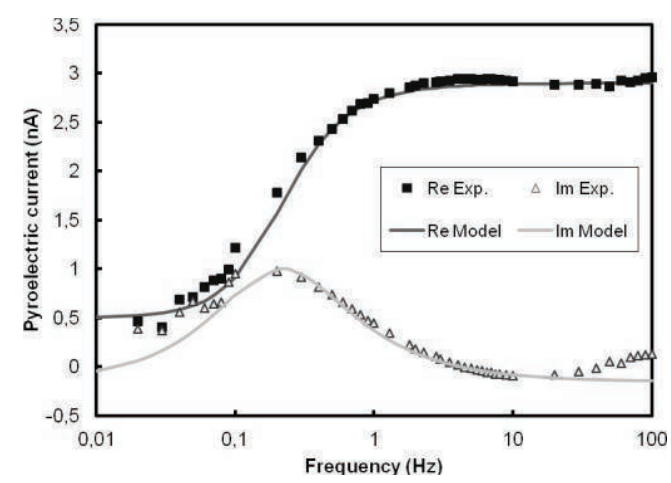

Fig. 3. Pyroelectric current spectrum of a PZT plate in comparison to a fit to eq. (3).

Our results demonstrate that the simple model in Ref. 5 can be successfully applied to embedded piezoelectric transducers.

\section{Thermal Pulse Method}

First thermal pulse measurements were performed with the PZT plate and the MFC actuator.

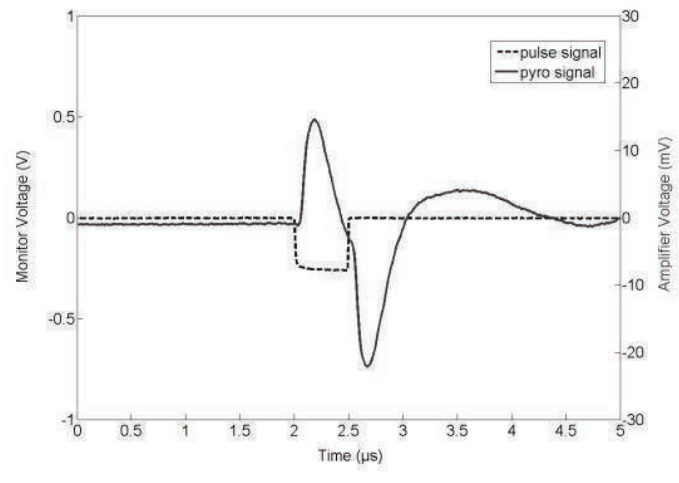

(a)

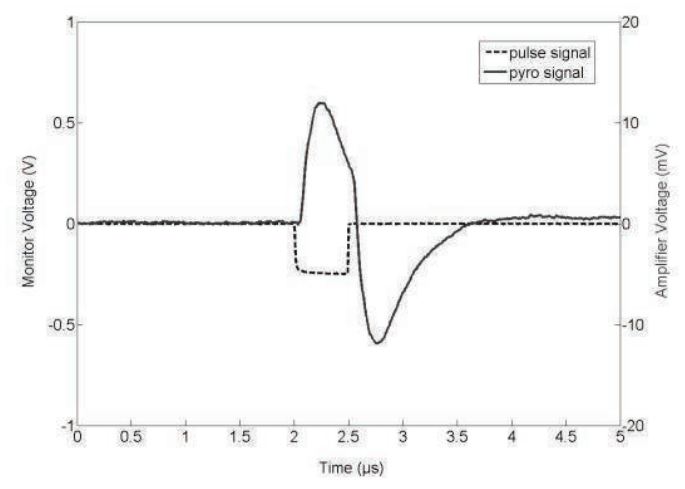

(b)

Fig. 4. Time dependence of the monitor voltage of the laser diode driver (scale of $-20 \mathrm{~A} / \mathrm{V}$ ) and the output voltage of the current amplifier $\left(10^{3} \mathrm{~V} / \mathrm{A}, \mathrm{DC}\right.$ coupling) for (a) the PZT plate and (b) the MFC actuator.

Fig. 4 illustrates the time dependence of the pyroelectric current for both samples. It shows the output voltage of the current amplifier in correspondence to the monitor voltage of the laser diode driver. The latter is proportional to the diode current and thus to the optical output power of the diode. Shortly after the beginning of the laser pulse the pyroelectric current rises. The zero crossing of the current indicates the temperature maximum of the sample. Cooling down to room temperature is characterized by a negative current because the pyroelectric current is proportional to the time derivative of the temperature (cf. eq. (1)).

Even for the low diode current of $5 \mathrm{~A}$ (corresponds to $12.5 \mathrm{~W}$ ) a relatively high pyroelectric signal was obtained. The higher heat output of pulsed laser diodes is one advantage of the pulse method in comparison to LIMM. This leads to higher signal levels which is beneficial in regard to embedded piezoceramics. The second advantage is the shorter measuring time which is important for quality control in mass production.

Further analysis of the pulse measurements is work in progress. 


\section{Conclusions}

We have demonstrated that the thermal wave method is a promising non-destructive tool to evaluate the polarization state of integrated piezoelectric transducers. The use of thermal pulses enables a higher signal output and a shorter measuring time. The next step is to Fourier-transform the time signal in order to apply the presented models in the frequency domain.

\section{Acknowledgement}

This research is supported by the Deutsche Forschungsgemeinschaft (DFG) in context of the Collaborative Research Centre/Transregio 39 PT-PIESA, subproject C8.

\section{References}

[1] A. Mellinger et al., Zerstörungsfreie Tomographie von Raumladungs- und Polarisationsverteilungen mittels Wärmepulsen (Non-destructive Spacecharge and Polarization Tomography with Thermal Pulses)., Technisches Messen 74, 437444 (2007); doi: 10.1524/teme.2007.74.9.437

[2] S.B. Lang, D.K. Das-Gupta, Laser-intensitymodulation method, a technique for determination of spatial distributions of polarization and space charge in polymer electrets, Journal of Applied Physics 59, 2151-2160 (1986); doi: $10.1063 / 1.336352$

[3] R.E. Collins, Measurement of charge distribution in electrets, Review of Scientific Instruments 48, 83-91 (1977); doi: 10.1063/1.1134854

[4] M. Flössel, S. Gebhardt, A. Schönecker, A. Michaelis, Development of a novel sensoractuator-module with ceramic multilayer technology, Journal of Ceramic Science and Technology 1, 55-58 (2010); doi: $10.4416 /$ JCST2010-00010

[5] S. Bauer, B. Ploss, A method for the measurement of the thermal, dielectric, and pyroelectric properties of thin films and their applications for integrated heat sensors, Journal of Applied Physics 68, 6361-6367 (1990); doi: $10.1063 / 1.346882$

[6] G. Suchaneck, A. Eydam, G. Gerlach, A laser intensity modulation method for the evaluation of the polarization state of embedded piezoceramics, Ferroelectrics 453, 127-132 (2013); doi: 10.1080/00150193.2013.842423

[7] A. Eydam et al., Polarization characterization of PZT disks and of embedded PZT plates by thermal wave methods, AIP Conference Proceeding 1627, 31-36 (2014); doi: $10.1063 / 1.4901653$ 